\title{
Percepciones de la población LGBT sobre la formulación de la política pública local
}

\author{
Daniela Jiménez* \\ (danielajimenez2011@hotmail.com)
}

\author{
Melissa Quiroga Mery ${ }^{* *}$ \\ (melymery@hotmail.com)
}

Artículo de investigación recibido el 26/11/2015 y aprobado el 19/05/2016.

Cómo citar este artículo:

JIMÉNEZ, Daniela y QUIROGA MERY, Melissa (2016). "Percepciones de la población LGBT sobre la formulación de la política pública local”. En: Transpasando Fronteras, Núm. 10, pp. 27-55. Cali, Colombia: Centro de Estudios Interdisciplinarios, Jurídicos, Sociales y Humanistas (CIES), Facultad de Derecho y Ciencias Sociales, Universidad Icesi.

\section{Resumen}

Este artículo presenta los hallazgos de un proyecto investigativo en torno a la formulación de la política pública para la población LGBT local entre septiembre y noviembre de 2013. De esta forma se pretendió indagar acerca de las percepciones de la población LGBT sobre dicha política pública, la cual se ha desarrollado entre manifestaciones de resistencia de aquellos directamente afectados como de acciones institucionales para ejecutar su formulación.

Palabras clave:

Población LGBT, política pública local, estudio de percepciones.

* $\quad$ Estudiante de Ciencia política de la Universidad Icesi (Cali, Colombia).

** Estudiante de Sociología y Ciencia Política de la Universidad Icesi (Cali, Colombia). 
"Me cierran la puerta y yo le meto el pie"-Andrea

"Yo soy un hombre homosexual que representa a una entidad llamada academia”-Andrés Castelar

"Nosotros LGBTI somos fragmentarios y fractales... ojala la gente pudiera ver que quien les dice que no venga a la formación, muchas veces se ha capacitado con recursos de la financiación internacional"-Mao Garcés

"Como gay se puede incidir sobre todo lo relacionado con la Población LGBTI y hace falta una mejor comunicación para poder persuadir."-Hebert Pérez

"Sino se hace nada van a sacar lo que se les venga en gana, van a sacar una copia de lo de Buga, Bogotá y de lo del Valle, una recopilación de eso (...) Yo ya estoy descalificándola, el proceso no ha sido el correcto, va a seguir siendo una politica de pacotilla."'- Revista Ambición

\section{Introducción}

La política pública ha sido señalada como uno de los mecanismos de acción e inclusión social estatal más efectivos, y ampliamente utilizados a lo largo de los diferentes regímenes democráticos del mundo durante las últimas décadas del siglo anterior y del presente. Desde el Estado Benefactor hasta la política actual, suplir las necesidades de los diferentes actores civiles se ha convertido en un requerimiento de primer orden dentro de la agenda estatal. En efecto, ha surgido como aquella herramienta destinada a facilitar y concretar la acción estatal frente a las demandas sociales de ciertos sectores, donde radican problemáticas específicas. Los bienes y servicios implícitos dentro de este ejercicio estatal han sido dirigidos a grupos principalmente considerados como vulnerables-sin dejar de lado otros sectores de la sociedad con otro tipo de demandas-. En el caso colombiano nos encontramos con varios sectores como los afrodescendientes, indígenas, mujeres, personas discapacitadas, víctimas del conflicto armado, desplazados y durante los últimos diez años el sector/comunidad LGBTI, en los cuales converge la vulneración de sus derechos. La política pública entendida como el conjunto de iniciativas, decisiones y acciones del 
régimen político frente a situaciones socialmente problemáticas (Vargas, 2007:85 en Arroyave 2011:96) se perfila en el contexto colombiano, como una de las alternativas de solución de problemas más incluyente y participativa. Sin embargo, Arroyave (2011) identifica tres retos y dificultades en las políticas públicas del país. El primero de ellos se refiere al desconocimiento que tienen los gobernantes sobre las políticas que van a aplicar, porque se limitan a delegar a un consultor y/o contratista el deber de la formulación y el estudio, quedándose sin conocer el panorama completo de la problemática. El segundo, refleja la situación vigente de las políticas públicas formuladas en el Consejo, el Congreso y la Asamblea, que si bien promulgan un componente de horizontalidad y alta participación de las comunidades, fallan rotundamente en la vinculación de estas últimas, de ahí que la formulación sea solo un "proceso simbólico de legitimación donde se vende la idea de inclusión y empoderamiento, construcción horizontal y conjunta de las políticas entre las comunidades, el Gobierno y los grupos de interés, como esfuerzo colectivo para responder a demandas insatisfechas" (Arroyave 2011:101).Y el tercero, es el triángulo de hierro donde se concentra el poder de funcionarios, empresarios y políticos, quienes en aras de buscar beneficios, las políticas formuladas terminan siendo el resultado de intereses particulares. Estos últimos terminan excluyendo los intereses y las demandas de la sociedad civil para la que estaba dirigida la política pública, aquella que con disímiles intereses intentan crear un espacio de mediación, en el cual se logren llegar a acuerdos que solucionen objetivos específicos, teniendo en cuenta insumos, recursos, contexto legal-político, y las reacciones que la misma pueda generar en la comunidad.

Lamentablemente, en el país nos encontramos con un modelo de políticas públicas que pregonan ser bottom-up, donde se supone que se "posibilita la inserción de los actores sociales quienes en un origen movilizaron la política pública (...) se pretende partir del núcleo del problema para construir así la solución de manera paulatina” (Roth, 2002 en Mejía, 2012:149) pero en realidad se refleja una mínima participación de los actores principales, dicha ciudadanía a la que va dirigida la política pública, ergo los resultados esperados no son significativos. Aún así, se siguen llevando a cabo diferentes proyectos a lo largo de todo el territorio nacional en pro de la formulación de políticas públicas, bajo el discurso de un país más democrático e inclusivo, uno de ellos la actual gestión de la política pública para la población LGBTI de la ciudad. Proceso al cual, a través de un 
ejercicio de carácter cualitativo-etnográfico le hemos seguido la pista y con el cual en aras del entendimiento, veeduría y control del proceso, hemos observado como al igual que en otras políticas públicas, inevitablemente se ha puesto "en tela de juicio los diseñadores de los programas o proyectos, las personas a cargo de la supervisión de las operaciones, y los mismos operadores.(...) [donde] están en juego aspectos como la reputación de las personas, sus prospectos de promoción profesionales o simplemente la continuación de actividades que les aseguren sus ingresos" (Jacques Mérat, 2009 :8), que han cambiado los rumbos de la formulación de la política y han desembocado en el disgusto de cierta parte de la población LGBTI, conocedora del proceso en cuanto a la metodología del proyecto, de su desarrollo, de la manera en que se ha trabajado la formulación de acuerdo a intereses políticos, la relevancia que se le ha dado y el trato que han recibido las personas a las que está dirigida el proyecto.

Estas son algunos de los hallazgos resultantes del proyecto investigativo en torno a la formulación de la política pública para la población LGBTI local en el periodo comprendido entre septiembre hasta el mes de noviembre de 2013, el cual pretendió indagar acerca de las percepciones de la población $\mathrm{LGBT}^{1}$ sobre dicha política pública y las intervenciones de los participantes están previamente autorizadas. Durante los tres últimos meses hemos atestiguado este proceso, el cual se ha desarrollado entre manifestaciones de resistencia de aquellos directamente afectados por la política pública y acciones institucionales para ejecutar la formulación, siendo requerida "una opción que ofrezca resultados mensurables pero que también se preocupe por el proceso político y democrático bajo el cual se define la necesidad de dichos resultados" (Cano, 2009: 42). Así, este ejercicio de veeduría del cumplimiento de las acciones efectivas, han generado tanto divergencias como consolidación de redes de apoyo entre los mismos actores del sector. A continuación daremos cuenta de nuestro proceso investigativo, producto del conocimiento adquirido en el curso de técnicas etnográficas y cualitativas, en donde primero realizaremos un breve esbozo a cerca del contexto de la política pública para la población LGBT a nivel nacional y depar-

1 La sigla utilizada para referirse a esta población ha sido ampliamente discutida en el ámbito académico llegándose a añadir paulatinamente una letra más, de acuerdo a las diferentes identidades y orientaciones sexuales: LGBT, LGBTI, LGBTIQA, LGBTPTTQQIIAA+ y TBLG. Sin embargo, los participantes de esta etapa de la investigación están contemplados bajo la primera denominación. 
tamental, posteriormente nos enfocaremos en describir el proceso y las impresiones de los participantes sobre la municipal, escenario en el que confluyen descripciones anecdóticas y opiniones contradictorias sobre el mismo. Luego, de manera paralela daremos cuenta de los interesantes hallazgos y el análisis de los mismos, y finalmente haremos el cierre con las decisivas conclusiones que obtuvimos de ellos.

\section{Esbozos sobre la política pública LGBT en el país}

La Constitución vigente, reafirmó el papel gestor y garante del Estado sobre los derechos y deberes de los ciudadanos. Se empezaron a gestar diferentes políticas en torno a ciertos grupos como los afrodescendientes e indígenas, quienes obtuvieron un trato con enfoque diferencial por parte del Estado, ese mismo al que hoy también apelan grupos como el sector/comunidad LGBTI, pues se observa que han sido vulnerados históricamente, afirmando tener necesidades todavía sin satisfacer por el Estado colombiano. El proceso para el reconocimiento de este grupo/sector ha sido complejo, diferentes organizaciones, activistas y personajes-no solo del sector- han tenido que luchar arduamente para contar con el poco reconocimiento que tienen. Paulatinamente han alcanzado logros, tales como la no discriminación por la orientación sexual, el derecho a la seguridad social de parejas del mismo sexo, libre desarrollo de la personalidad en diferentes espacios públicos, derechos patrimoniales, derecho de alimentación de parejas del mismo sexo, cambio de sexo y de nombre, posibilidad de heredar los bienes de la pareja del mismo sexo, entre otros (Sotomayor, 2013, p.95-96). Estos reconocimientos y derechos han conducido al sector a demandar soluciones a problemáticas a nivel nacional, departamental y municipal.

La construcción de políticas públicas $\mathrm{LGBT}^{2}$ en el país ha sido generada por la misma presión del movimiento sobre las autoridades, en donde Bogotá y Valle del Cauca toman la vocería. En una entrevista realizada a Diana Navarro (vocera de la comunidad LGBT en Bogotá) por Buriticá (2010) se señalaba que debido al esfuerzo del propio sector fue que se lograron concretar acercamientos con las autoridades, donde se indagó por el establecimiento de acciones que atendieran las necesidades y el manejo que debía dárseles a

2 Encontramos que en ciudades como Bogotá y Medellín se omite la I que corresponde a la categoría de personas intersexuales, lo cual deja el interrogante a cerca del nivel de inclusión de la política pública empezando por el nombre de la misma. 
las mismas, en una lógica de abajo hacia arriba. Si no hubiera sido gracias a la presión del sector en cuestión, la construcción de la política pública probablemente hubiera tomado más tiempo en tener forma y en llegar a la agenda política capitalina

Valle del Cauca fue el primero en tomar la iniciativa, esto durante el gobierno de Angelino Garzón en el 2006, donde a través del Acuerdo Social de Voluntades entre la Gobernación del departamento y catorce diversas organizaciones, activistas, líderes y liderezas LGBTI se marcó una pauta para la construcción de la política departamental dirigida a la población LGBTI. En este se fortaleció el compromiso departamental por una mayor inclusión de las poblaciones vulnerados, con la ayuda y presencia de los futuros beneficiados de la política, quienes se comprometieron junto a la gobernación del Valle a una pronta formulación y ejecución de una política pública, redireccionando así la agenda política departamental. A partir de ese momento empieza a tener un enfoque de mayor visibilización y satisfacción de demandas del sector, pues confluyeron en diez compromisos bases. Si bien persistió la presión y el impulso por parte de miembros y organizaciones del sector en el 2007 y los siguientes dos años para la formulación de la política pública, sólo fue en el 2010 a través de la Secretaria de Equidad de Género y el gobierno de Francisco José Lourido cuando se inició la construcción de los lineamientos de la política pública LGBTI, que desembocó en el siguiente año con la Ordenanza 399 del $2011^{3}$. La cual actualmente se ejecuta a través de la socialización, sensibilización y caracterización del sector del proyecto de "Promoción de las políticas públicas para Equidad de Mujeres Vallecaucanas y de los sectores sociales LGBTI".

En el caso de Bogotá, durante el gobierno de Luis Eduardo Garzón se expidió el Decreto 608 de 2007 en el que se establecieron los lineamientos de la política pública distrital para la garantía de los derechos del sector en cuestión, y posteriormente en el Acuerdo 126 de 2009 se estipularon finalmente los objetivos e implicaciones de tal política pública. Actualmente se encuentra en ejercicio y tras las reformas hechas por el actual alcalde, se ha venido implementado una serie de medidas incluyentes que van desde la creación de

3 Existe una dualidad y confusión con respecto al número de la ordenanza. En la página oficial de la Gobernación del Valle esta disponible el documento de la Ordenanza 399 del veintiocho de Diciembre de 2011, sin embargo también existe un comunicado oficial en donde se hace referencia a la Ordenanza 490 del primero de Diciembre de 2011 que también es referenciada en otros sitios web que defienden los derechos de las personas LGBTI. 
una Subdirección LGBT dentro de la Secretaría de Integración Social del Distrito Mayor hasta diferentes actividades culturales que han dado cuenta de un incremento en la participación e inclusión del sector en la agenda municipal.

Buga y Medellín hacen parte de la lista de ciudades que ya pasaron por la formulación de la política pública y actualmente se encuentran gestando la aprobación y ejecución de las mismas. "Una constelación de diversidades" es para la pequeña ciudad de Buga un gran paso la protección de los derechos de las personas LGBTI y la inclusión de estas en diferentes ámbitos sociales, a pesar de que la aprobación por el Concejo y su establecimiento en el Acuerdo 058 de 2013 todavía tiene un largo camino por recorrer empezando por su eficaz socialización y divulgación para lograr los objetivos de la política, pues su desarrollo ha girado en torno a la promoción de actividades como desfiles y reinados, impulsadas y desarrolladas por los gestores del proyecto. Medellín por su parte logró en el 2011 la consolidación del Acuerdo 08 de 2011,al establecer una política que se encargara de la especial protección de la población, el respeto a sus derechos y el reconocimiento de la identidad de género y orientación sexual. La política "pretende que la Administración Municipal materialice programas dirigidos a la transformación de los imaginarios y las situaciones que generan discriminación y exclusión de personas pertenecientes a esta población. Además, que se destinen recursos económicos para la ejecución de proyectos y medidas que logren el respeto de la dignidad y los derechos de este importante segmento poblacional, así como la participación en las instancias de decisión y seguimiento a la Política Pública"4. Si bien es reconocida ampliamente en el país por la cobertura que tiene en los diferentes temas que afectan a la población tales como salud, trabajo digno, educación y seguridad, que se deben en parte a la creación de tres entidades encargadas de hacer el seguimiento, evaluación y veeduría de la política, el Comité Municipal de Diversidad Sexual e Identidades de Género, el Consejo Consultivo, y el Centro para la Diversidad Sexual y de Género, también es reconocida por una horizontalidad relegada a favores políticos. La política pública de Medellín es el

4 Ver http://www.caracol.com.co/noticias/regionales/medellin-es-la-primera-ciudad-en-el-pais-conpolitica-publica-para-el-sector-lgbt/20110416/nota/1456385.aspx Consultado el 18 de noviembre de 2013 


\begin{abstract}
"Resultado de un compromiso electoral entre sus líderes en la ciudad y el alcalde del momento (Sergio Fajardo Valderrama). En [este] (...) proceso se prioriza el pago de favores politicos ante la necesidad de reconocer y garantizar un derecho a las minorías sexuales de la ciudad, a tiempo que se invisibilizan las bases de esta comunidad para la identificación, construcción y ejecución de la política, y por lo general se asignan a consultores y a algunos integrantes de la población LGBT amigos de la administración, pero que aún están lejos de una transformación cultural que solucione los problemas a tratar" (Arroyave, 2011:99)
\end{abstract}

A nivel nacional nos encontramos con el Decreto 4530 de 2008, que da pie para la gestión del Ministerio del Interior y Justicia en la construcción de la política pública LGBT de la mano de los miembros del sector, organizaciones sociales y activistas de los lineamientos de la política pública nacional, la cual ya lleva tres años formulándose a través de la Dirección de Asuntos Indígenas, Rom y Minorías. Su formulación se ha venido gestando por medio de la colaboración de organizaciones e identidades relevantes dentro de la población, la creación de mesas de trabajo a lo largo del territorio nacional, mesas de casos urgentes para tratar con la población vulnerada, el diseño de planes y programas dirigidos a la protección y defensa de los derechos de la población, entre otros.

Según lo dicho, podríamos hablar de la relevancia que tienen el grupo en cuestión para la formulación de la política, sin embargo, posteriormente debido a cuestiones legales y burocráticas, van perdiendo participación e incidencia en el proceso en que converge en una política top-down ${ }^{5}$. En este sentido, la iniciativa no se gesta hasta que las organizaciones del sector, probablemente apoyadas por organizaciones internacionales, se movilizan a exigir su inclusión en los diferentes escenarios políticos, que se traducen en la creación y/o modificación de Secretarías, Comisiones, Consejos, Departamentos, Centros, Divisiones, Direcciones especializados en este grupo, tal y como la Secretaría de Equidad de Género, Diversidad Sexual e Identidad de Género, la Secretaria de Mujer, Género y Diversidad Sexual y la Dirección de Diversidad Sexual, Dirección de Asuntos

5 "Trae consigo el clásico trabajo de la administración pública que se desarrolla de arriba hacia abajo, o del centro a la periferia; aquí prima la jerarquía y establece una diferencia entre la formulación, la toma de decisiones y la implementación" (Roth, 2002 en Mejía, 2012:149) 
Indígenas, Rom y Minorías, Subdirección LGBT, que en la mayoría de los casos no son dirigidas y/o altamente influenciadas por personas del sector haciendo que su voz se vea menguada por la misma burocracia encargada de su protección y defensa.

\section{Diplomado y percepciones: La investigación}

La amplia convocatoria para la formulación de la política pública para la población LGBTI llegó a nuestras manos, y tras el fallido intento de una investigación dirigida únicamente a los mecanismos de participación ciudadana hacia una sola población, optamos por extender nuestro campo e indagar la coyuntura política de dicha formulación. La convocatoria presenta un Diplomado de puertas abiertas creado para la formulación de la política pública para la población LGBTI que durante su transcurso fue llamado de diferentes formas tales como Diplomado (en, de, para la formulación de ) política pública: LGBTI, de Derechos Humanos en Diversidad Sexual, Diversidad Sexual e Identidad de Género, para personas en condición de Diversidad Sexual e Identidad de Género y finalmente -como posiblemente sea llamada en el Acuerdo de la política pública- para personas en contexto de Diversidad Sexual e Identidades de Género. Esta se constituye entonces como un espacio en el que se pretende concretar las repuestas del gobierno ante las diversas expectativas y demandas que la población LGBT manifiesta, además de continuar con la manifestación de quejas, necesidades, activismo y participación ciudadana de manera más formal por parte de voceros de fundaciones, activistas independientes, no activistas, comerciantes enfocados en la diversión nocturna, instituciones estatales, promotores culturales, académicos, diseñadores, administradores de prostíbulos, entre otro/ as. Lamentablemente no pudimos observar el proceso desde el comienzo, empezamos a asistir al diplomado y a desarrollar el trabajo de campo cuando ya había avanzado en sus primeros módulos el Diplomado. Aun así pudimos ponernos al tanto de las discusiones y desacuerdos de este escenario, y a partir de ahí se empezó a trabajar.

Dentro de los puntos importantes que cabe destacar están: la masiva asistencia inicial del diplomado, la disminución de la participación y asistencia conforme pasaban las clases, las no tan diversas posturas de los asistentes y la baja asistencia de personas pertenecientes al sector o por lo menos diversas, ya fuera en cuanto a orientación sexual y/o identidad de género en contraste con la asistencia de personas heterosexuales. Ante este descenso de la 
participación, la posibilidad de obtener multiplicidad de visiones de la población sobre el proceso se redujo y si bien es posible que las razones que acompañaron este descenso se vean reflejadas en los cuestionamientos sobre el proceso de los pocos asistentes del sector, como el manejo y la legitimidad en la gestión de la política pública, no tenemos garantía de que nuestra investigación no haya sido ampliamente influenciada e incluso sesgada por las reducidas percepciones. Factor importante para la determinación de nuestro análisis y conclusiones finales, hiladas principalmente por las conversaciones y entrevistas de algunos asistentes y empleados de la organización en cuestión.

Esta investigación se desarrolló aproximadamente en tres meses y medio y es el resultado del proyecto investigativo del curso transdisciplinar de Técnicas cualitativas y etnográficas, donde pretendimos rastrear percepciones del sector/comunidad LGBT sobre la política pública local. Las trascendentales herramientas de investigación como los diarios de campo, la observación participante y descripciones etnográficas en la investigación, sumado al seguimiento del material documental trasmitido por el Instituto Prospectiva, entrevistas a los actores principales y visitas a otros espacios, constituyeron la columna vertebral de la investigación y la forma en que se llevó a cabo. La investigación no ha concluido, al contrario, es tan solo la primera parte del intento de comprensión de la políticas de inclusión colombianas, su aplicabilidad, viabilidad, desarrollo, ejecución, bajo una idea democrática de participación e inclusión que pretenden abarcar a esta vulnerada, excluida, marginada y discriminada población. De igual forma, este es un primer paso de veeduría ciudadana a una política pública que no solo concierne a la población LGBTI sino que también converge en los intereses generales de la sociedad, en este caso la caleña, de la que hacemos parte.

\section{Dilemas y percepciones de la formulación de la política pública local LGBTI}

El nombre de la política pública es además de la contratación uno de los asuntos más cuestionados y polémicos del proceso. Tal y como ya lo mencionamos, la referencia e intercambiabilidad de palabras y nombres para hacer alusión a la política pública ha generado disgustos y confrontaciones. El Instituto Prospectiva Innovación y Gestión de Conocimiento designado para la gestionar el Diplomado y asimismo la formulación de la política pública, a lo largo del diplomado hizo uso indistintamente de diferentes nombres 
para referirse a política pública. Además de los ya aludidos- política pública LGBTI, política pública de Diversidad Sexual e Identidad de Género, política pública para personas en condición de Diversidad Sexual e Identidad de Género y política pública para personas en contexto de Diversidad Sexual e Identidades de Género- el nombre más utilizado e incluso referido en el mismo contrato interadministrativo fue el de "Política Pública para personas en condición de Diversidad Sexual y de Género". Este último fue sin duda el más controversial y debatido. Por un lado, varios de los manifestantes principalmente pertenecientes al sector se mostraron inconformes y disgustados con el término -condición-, ya que para ellos dicha palabra tenía una connotación de inferioridad y/o enfermedad, que en caso de ser utilizada para referirse y ejecutar la política, perpetuaría la discriminación que afecta a la población, la cual muy probablemente continuaría siendo referida como un grupo marginal, inferior, anormal y enfermo, el cual debe ser tratado, combatido y/o eliminado. También el uso de la palabra LGBTI resultó no ser del todo aceptada para su uso dentro del nombre y referencia de la política, principalmente por el componente político, y en algunos casos, discriminatorio contenido en la pragmaticidad de lo lgbetesco. El uso de las Diversidades Sexuales e Identidades de Género fue al parecer uno de los nombres más aceptados, y según el último borrador del Acuerdo de la política podría ser la forma en que sería referenciada la política, que podría llamarse "Política pública para el reconocimiento de las personas en contexto ${ }^{6}$ de diversidad sexual e identidades de género y la protección, restablecimiento, atención y la garantía de derechos de las personas lesbianas, gays, bisexuales, trans e intersexuales (LGBTI) del Municipio de Santiago de Cali".

La contratación es el punto de partida para el inicio de la formulación de la Política Pública del sector, pero también el inicio de procesos paralelos que conforman el proyecto: Diplomado en Política Pública y Derechos Humanos en Diversidad Sexual del municipio, la construcción de las mesas de trabajo, la caracterización, el plan Decenal y el proyecto de Acuerdo. Una vez fue contratado el Instituto Prospectiva, Innovación, se empezó a gestar la formulación de la política a través de la creación de un Diplomado diseñado para la construcción de la política conjunto a sus asistentes, independientemente de su

$6 \quad$ ¿Es la palabra contexto la más adecuada para hacer referencia a esta población teniendo en cuenta las críticas hechas por la misma población a cerca de la connotación negativa de la palabra? 
pertenencia a la población o no. Para ello el Instituto realizó una amplia convocatoria, la cual entidades como la Revista Ambición ${ }^{7}$ cataloga de incorrecta, pues esta argumenta que el llamado debió ser dirigido por la Secretaría. A pesar de la masiva asistencia de organizaciones, líderes, liderezas, activistas independientes, miembros del sector, académicos y personas heterosexuales, diferentes testimonios de participantes del Diplomado que siguieron todo el proceso de la construcción de la Política Pública, advierten que si bien hubo un número considerable de asistencia por parte del sector LGBTI, aproximadamente un ochenta por ciento de los asistentes, de acuerdo a lo que lograban observar, no hacían parte del mismo y sólo estaban interesados en el aprendizaje de la formulación de una política pública y/o obtención de un cartón. Así, al ser una convocatoria abierta, se permitió que personas que desconocían la realidad del sector hicieran parte de manera igualitaria en el proceso de la formulación.

Gran parte de la discordia que se generó en torno a la inclusión de personas heterosexuales y/o enajenadas por completo a las realidades LGBTI, radicó en la inconformidad del sector por involucrar, tener en cuenta y en algunos casos, dialogar con percepciones contradictorias a las iniciativas incluyentes y participativas de la Política Pública. A pesar de ello, la asistencia de estas personas no menguo por las inconformidades, incluso siguieron representando una mayoría dentro del Diplomado y podría decirse que aletargaron la formulación. Por esta razón fue necesario informar a todos sobre las realidades del sector, dar cuenta de qué estaba haciendo cada organización, qué necesidades presentaban, qué demandas tenían, qué derechos estaban siendo vulnerados, y en efecto, ello se tradujo a empezar desde cero, para suplir la asimetría de conocimiento, no solo por parte de las personas heterosexuales sino también por aquellas personas que presentaban cierto "desnivel" de conocimiento/educación frente a otras.

\footnotetext{
"Cuando se abre el diplomado a personas heterosexuales no previeron de que pasara lo que pasó, que llegan las personas incautas pocamente interesadas en un proceso politico y que llegan a decir bueno... que eso de LGBT que yo no sé que es... yo vengo a la política pública porque es una política y es un diplomado ¿me explican? Y entonces uno aquí sentado (...) Salieron a decir que
}

7 "Revista de Bolsillo de distribución gratuita dirigida a la comunidad LGBTI+H del suroccidente Colombiano y el eje cafetero"(http://issuu.com/revistaambicion/docs/revista_ambicion_segunda_edicion) 
era un diplomado pa' todo el mundo de 140 personas, gente despistada que se dedicó a ir alli a que le dieran el refrigerio, de salir ahí a seguirnos tratando de freaks ¿y por qué? Porque tiene derecho, porque es ciudadano. No arrancaron de cero, no hicieron un paseo, no hicieron una fiesta de integración. Entonces en la tercera o cuarta semana, lo que dijo alguien en una reunión: yo veía un muchachito que él era muy mariquita muy amanerado... o sea no era como los otros amiguitos que si eran normales, el era muy amanerado y ese muchachito se quiso suicidar y esta persona lo dice delante de activistas, mujeres trans, mejor dicho...y ustedes saben pues que esas sensibilidades del lenguaje aquí están a flor de piel" (Entrevista a Andrés Felipe Castelar, 12 de Octubre 2013)

Lo anterior, resulta ser un incentivo para reforzar la inconformidad por parte del sector LGBTI frente a la idoneidad del Instituto Prospectiva, haciéndose hincapié en cuestionamientos como: ¿Realmente un proceso dirigido a la comunidad LGBTI debe ser encabezado y ampliamente influenciado por personas ajenas al sector, como personas heterosexuales? Interrogante que señala la importancia de legitimar a los actores- más en un sector donde el carácter identitario se constituye como su fundamento de agrupación. Este tipo de cuestionamientos fueron reiterativos en el análisis que los participantes del Diplomado hacían sobre Prospectiva, alimentando un poco nuestra comprensión sobre cómo debe realizarse una política pública, de ahí que en una entrevista realizada a un representante de la Revista Ambición, perteneciente al sector, señalara que

\footnotetext{
"La selección de prospectiva en este proceso no es comprensible. Hasta el momento no hemos encontrado una razón de por qué escogieron a Prospectiva para realizar este proceso. Propsectiva no tiene experiencia con comunidad $L G B T$, no tiene ni idea cómo se trabaja con la comunidad LGBT. ¿Cómo lo escogieron? yo no sé. No hubo una convocatoria real. Esto es un proceso público y exige convocatoria, no la hubo. Cómo es posible que las sociedad civil que está trabajando con comunidad LGBTI durante tanto tiempo no se de cuenta de una convocatoria de estas, cuando algunas de estas organizaciones tienen todas las capacidades para realizar estos proyectos (...)
}

Hubo una asistencia de cómo 90, muchísima gente pero de esos 90 el 70\% no hacia parte del sector y en alguna reunión le dije yo: ¿qué? ¿Vamos a hacer una Política pública de negritudes con 2 negros y 50 blancos? ¿Qué tiene eso de in- 
cluyente? Si se permite el acceso de personas que no hacen parte del sector pero ¿conoce del tema? ¿Conoce el sector? ¿Ha trabajado con el sector? ¿Conoce cada una de las identidades? ¿Sabe usted que significa la I que está ahi? ¿Sabe cuántas identidades hacen parte de esa T? no la conoce. Entonces, usted no conoce el problema de fondo, ahí es donde está el asunto de la participación hetero. Aunque lo hetero también hace parte de las diversidades sexuales" (Entrevista a Representante de la Revista Ambición, 17 de noviembre 2013).

La idoneidad de Prospectiva es cuestionada desde su contratación. Cuando recién se hizo la convocatoria de invitación a la construcción de la Política Pública el abanico de preguntas versaban sobre: ¿Por qué el Instituto de Prospectiva de la Universidad del Valle había sido elegido para tal proyecto?, ¿Por qué existiendo organizaciones reconocidas del sector conocedoras del tema, se eligió a Prospectiva que es un agente ajeno al sector? ¿Cuál es la experiencia de Prospectiva en temas de Diversidad Sexual y de Género? ¿Es suficiente con su trayectoria en otros proyectos municipales? y ¿Dónde queda el componente de diversidad dentro de su ejercicio? Estos y muchos otros interrogantes no solo pasaron por la cabeza de los participantes del Diplomado sino también por la de nosotras, que tras la apelación por una explicación que señalara los motivos de la contratación, los argumentos esbozados aún son confusos. Hay varias explicaciones de ello, es decir, de elegir a Prospectiva como la institución más idónea para el proyecto, entre ellas una contradictoria, pues un funcionario de Prospectiva manifestó que, en primer lugar, se hizo la convocatoria a diferentes universidades como la Universidad Icesi, Autónoma de Occidente y la Universidad del Valle. Tras, el respectivo análisis de las propuestas se eligió a la Universidad del Valle y su Instituto Prospectiva por su experiencia, éxito y competitividad en la formulación de políticas públicas. Sin embargo, en otro momento de la entrevista no encontramos con otros pronunciamientos:

"sé que hay un concepto que se llama la autonomía administrativa que como tal la Secretaria de desarrollo territorial, sé que hizo una invitación a diferentes universidades de la ciudad y la propuesta que más cercana en términos de idoneidad, de costo y de cobertura social fue la de la Universidad del Valle, hasta ahi te puedo decir que es lo que yo sé. Digamos que la Universidad del Valle ha tenido un trabajo con el sector LGBTI ha sido amplio porque la Universidad del Valle es como una mano que tiene varios dedos 
tiene diferentes institutos de psicología, de género, de comunicaciones y el de Prospectiva, que es el encargado de agendar agendas de políticas públicas. Entonces con esa combinación uno ve una institución como la universidad del Valle, ve una institución de 65 años de reconocimiento nacional e internacional, creo que fueron esos los valores tenidos en cuenta para asignar un convenio interadministrativo, que es Estado contratando Estado, lo cual daba una garantía de transparencia y de manejo" (Entrevista a "Victor" funcionario de Prospectiva, 18 noviembre de 2013).

Ello se contrasta con la manifestación de otro de los miembros de Prospectiva, Hebert Pérez, miembro del comité académico, gay e impulsor del Diplomado, quien nos explicó la contratación de la siguiente manera:

"Yo supe a principios de año que la Secretaría de Desarrollo Territorial y Bienestar Social estaba buscando un proceso de política pública con la comunidad LGBTI, eso lo supe y luego quedó en silencio y luego me llamaron a ser parte del equipo. Ahi me di cuenta que había sido asignado al Instituto de Prospectiva, Innovación y Gestión y entonces hasta alli. Después de que han surgido toda esta serie de cuestionamientos por parte de algunas personas de la población LGBTI de Cali, de qué se hizo, de qué no, es un contrato administrativo, eso significa que es hecho a dedo. Un Contrato Interadministrativo es diferente a una licitación pública, en la cual se cumple un proceso, se licita y vienen aquellas personas que creen que pueden hacer eso, se presentan una serie de documentos que cumplan los requerimientos de la licitación, pues esperan que hagan el comité evaluador y decidan, no hubo una convocatoria... Pues si fue un contrato interadministrativo no creo que haya habido una convocatoria, entonces la segunda parte, en el contrato interadministrativo, la Universidad del Valle es idónea en muchas cosas, igual que el Icesi. Ellos tienen muchos contratos interadministrativos, entonces las empresas, el sector publico los buscan. Si legalmente yo no quiero hacer licitación y soy parte del sector público, yo tengo esta opción porque yo sé de su idoneidad para irme con usted pero yo le pido inicialmente una propuesta técnica, se cumplen una serie de requisitos, si cumple eso ya entra a trabajar. Eso va y viene, no es de un día para el otro, toma su tiempo y es también un proceso". (Entrevista a Herbert Pérez, 18 noviembre 2013). 
Por otro lado, el profesor Andrés Felipe Castelar nos expresó:

Hacen una convocatoria diplomado para crear la política pública municipal, a las dos semanas sale otro correo invitando a un ejercicio parecido pero departamental, de la cual aparentemente los lineamientos ya están hechos y están aprobados. (...) Se empezaron a hacer las reuniones de los diplomados con una agenda que en la cabeza de Prospectiva, era una agenda buena para empezar a producir los insumos de esas políticas públicas. [Pero] (...) era una agenda que ya existía, que no se revisó, una agenda en la que quienes la propusieron pensaron que era lo más útil para ustedes. (...) [Considero que se] ha tratado de hacer bien, me parece que el contrato con la universidad, pues contrato con quien tenía que contratar, el asunto de legitimidad es otro(...) Finalmente el Instituto de Prospectiva es un mercenario ¿si? Hacen el contrato porque además son el único que pueden contratar, porque ya han participado en otras formación de políticas públicas y yo no sé cómo les haya ido a ellos en la convocatoria, Yo no sé si ellos le escribieron a ellos y les dijeron que no (Entrevista Andrés Felipe Castelar, 12 octubre 2013)

Nos encontramos así, con la opinión de un funcionario de Prospectiva, Herbert Pérez, la Revista Ambición y Andrés Felipe Castelar, cuatro posturas diferentes que dejan un variopinto número de posibilidades para la formulación de la política pública local. El primero de ellos plantea que si bien hay una experticia en cuanto a conocimiento de realidades del sector y diferentes procesos con el mismo, las organizaciones caleñas LGBTI no están suficientemente capacitadas para dirigir un proyecto de tal talante. El segundo de ellos expresa que Prospectiva se ha ganado una distinción por la magnitud de proyectos ejecutados anteriormente y por las políticas públicas que ha direccionado, evaluado y fortalecido, aunque apunta falencias en cuanto a la profundidad y conocimiento que posee el Instituto sobre el tema LGBTI. Revista Ambición da fe de la capacidad de las diferentes organizaciones para hacer frente a la formulación de la Política Pública local, las cuales a través de una sinergia temporal lograrían dirigir el proyecto. Y finalmente, si bien Castelar reconoce el trabajo de Prospectiva y lo identifica como una institución apropiada para el proyecto también critica sus falencias durante el proceso de la formulación. De este modo, persiste la crítica y la pregunta sobre quién debería -o debió haberse encargado de- 
ejecutar el proyecto, pues si bien la metodología de la formulación de la Política Pública local intentó albergar un carácter participativo, la realidad es otra.

La metodología presenta una serie de incongruencias y problemas en su desarrollo debido a las inconformidades presentadas por los asistentes, que causaron un giro en la implementación de la misma. En el papel, la metodología contenía la aplicación de actividades tipo seminario-taller, que propiciaran un proceso de aprendizaje a través de las experiencias de los participantes, sumado al componente académico que suponían las clases del diplomado. Inicialmente los sábados y domingos de agosto y septiembre no fueron más que clases presenciales en donde se dictó un recorrido histórico de lo lgbtesco, las identidades de género, orientaciones sexuales y la fatigosa explicación de qué es ser gay/lesbiana/trans para cada uno de los asistentes del diplomado a sus compañeros heterosexuales.

\begin{abstract}
"Las personas que iban lo tomaban como una clase, dejen hablar al profesor, las preguntas al final, el refrigerio, profe que pena llegue tarde ¿Cuál es la tarea? (...) [Luego ellos] dijeron: vamos a hacer un proceso participativo, vamos a hacer un proceso consensuado entonces los asuntos de forma no se ven: ya nos van a habilitar el blog, nosotros les vamos a dar las actas jy eso dónde está? Dieron un papayaso para que inmediatamente empezaran a mostrar que ellos decian que iban a ser un proceso incluyente y lo incumplieron. Entonces lentamente se empezó a hacer el ejercicio de resistencia" (Entrevista a Andrés Felipe Castelar, 12 octubre 2013).
\end{abstract}

Una vez se empezó a desarrollar la metodología participativa, se observó un alto nivel de participación de los asistentes, quienes incluso se extendían en sus intervenciones y generaban mayor discusión, que en algunos casos dieron como resultado enfrentamiento de posturas y alegatos entre los mismos. Sin embargo, dichas intervenciones en muchas ocasiones fueron pasadas por alto, y más que un proceso de aprendizaje conjunto, se convirtió en un proceso renuente al cambio que supuso a largo plazo la deserción de muchos miembros del Diplomado. Lo anterior se vio expresado en situaciones como las propuestas de cancelación del contrato por parte de la Secretaría a Prospectiva, cambios de dinámicas del Diplomado como la suspensión del mismo durante dos sábados consecutivos, reconsideración de los lineamientos y del ente ejecutor del proceso, separación 
de las mesas respecto al diplomado y a la formulación de la política (este último si se efectuó), de ahí que Revista Ambición diera cuenta de la falta de interés de la institución por la inclusión de otros puntos de vista dentro del proceso, ante una actitud de negación por parte de uno de los integrantes de Prospectiva, quien tras indagar sobre la opinión a algunos participantes de las mesas sobre el afiche destinado a la difusión de la política, rechazó las propuestas y sugirió que el afiche no estaba presto a modificaciones.

\begin{abstract}
"El Diplomado que ellos planteaban era supuestamente participativo, pero su concepto de participativo era ponerte a hacer su trabajo de campo-se hace así, háganlo ustedes- Ellos pretendían que vos tuvieras que generar unos insumos, salir a la calle a hacer investigación para ellos. A fin de recoger sus propios insumos para hacer un producto, ni siquiera para generar un proceso" (Entrevista a Representante de la Revista Ambición, 17 de noviembre 2013).
\end{abstract}

En el caso de la participación en las mesas de trabajo, el número de estos fue muy reducido, pues aproximadamente solo diez personas participan, "representan" y discuten sobre las once temáticas y las siete mesas de: cultura, deporte, empleo, educación, competencias ciudadanas, salud y justicia. No obstante, la caída de la asistencia en los dos espacios se debió a: las inconformidades por la contratación, falencias de Prospectiva en el trato y lenguaje necesario para referirse a la población, desinterés del Instituto por ahondar en las vivencias que pretendían incluir en su metodología inicial, el horario, las condiciones de trabajo, el espacio, que en ocasiones se delimitaba en zonas alejadas de la universidad, la dinámica de las actividades, la falta de inclusión de otras perspectivas, comentarios y propuestas diferentes al de la institucionalidad y finalmente, la apatía e indiferencia de organizaciones relevantes del sector argumentando una postura crítica y deslegitimadora del proceso.

Teniendo lugar divergencias entre las organizaciones y activistas, acentuadas en una insistente negativa de ceder a cambios por parte de Prospectiva y de la Secretaría de Desarrollo Territorial y Bienestar Social, que posiblemente se quedan en la invitación participativa del ejercicio y no trascienden a la implementación y evaluación de las propuestas para reorientar la formulación de la política pública local. En dicho proceso, diferentes actores dieron origen al Ala Disidente o el Grupo de las Nueve, que se autodefine como 
"una tendencia autónoma de Organizaciones, Liderazgos, Líderes, Lideresas, Activistas, Base Social, Organizaciones y Defensores de Derechos Humanos LGBT de Cali. Es una voz y movilización social que ejerce el derecho a la oposición consagrado en la Constitución Política de Colombia y hace resistencia civil evidenciando las falencias, mala planificación, abordaje y lógicas perversas para el diseño, formulación, construcción e implementación de la política pública LGBT en Cali” (Grupo de las Nueve, 2013). Las fuertes críticas de este grupo se convirtieron en una traba, poniendo en entre dicho una vez tras otra el accionar de Prospectiva. Los comentarios hechos por este grupo fueron omitidos o refutados, a veces de manera reacia y cuestionable. Se debe reconocer que en algunas ocasiones el grupo hizo una participación agresiva, sin embargo, gran parte de sus críticas concordaban con el comportamiento y el desarrollo del proceso por parte de Prospectiva. Este grupo le dio un giro completo al Diplomado porque condujo a Prospectiva a esforzarse por recuperar su escenario y legitimidad dentro del proceso. Hicieron aflorar una serie de interrogantes como: ¿Son estas las personas, las encargadas de formular la política para personas tan sensibles y demandantes como lo es la comunidad/sector LGBTI? ¿Qué se debe esperar del proceso? sumado a que con la extensión de las discusiones, contratiempos, mala logística ¿En realidad, resultará siendo un proceso exitoso? ¿Será suficiente el tiempo para formular la política pública? Y si se logra ¿Luego, qué? ¿Quién se va encargar de liderarla y hacerla cumplir? Bajo un contexto en el que el seguimiento del tiempo señalado para su consecución, se convierte en un imperativo directo que condiciona el proceso.

Las mesas tomaron aproxidamente un mes en labores para construir la política pública. Como ya se mencionó, son alrededor de diez personas que las conforman, las cuales no se encuentran claramente definidas. Para este objetivo todos se reúnen en un mismo salón, en el que no se establece un orden en las intervenciones y no se desarrollan dinámicas que permitan escucharse atentamente entre sí. Uno de los fragmentos de los diarios de campo así lo confirman:

[Según el correo, la gente estaba citada para las 5:00 pm. El lugar en el que se celebraban las reuniones de estas mesas quedaba en uno de los lugares más recónditos de la Universidad, el antiguo Colegio de Las Marianitas. En efecto, estuve en el lugar a las 5:00 en punto y sólo se encontraba la persona encargada de registrar los nombres en cada sesión, tanto las del Diplomado 
como las de las Mesas. Alrededor de las 5:20 observé a algunos funcionarios del Instituto Prospectiva tomando un refrigerio en la cafetería. Se tenían destinados alrededor de cuatro salones señalados con letreros que indicaba si correspondía al escenario de educación, empleo, justicia, etc. Cuando regresé, el saldo de los asistentes lo conformábamos una persona del sector y yo. Esta persona eventualmente hacía comentarios sobre la poca asistencia pero ninguno de los funcionarios se percató de escucharla. El salón lo constituían 40 sillas, ubicadas paralelamente una de la otra, como en un salón de clases, diferente de lo que yo esperaba ver de unas mesas de trabajo. Alrededor de las 5:40 habia 12 personas, de las cuales más de la mitad eran representantes y funcionarios del Instituto Prospectiva, pero aún no se daba inicio. Mientras tanto, algunas personas hablaban separadamente, los funcionarios de Prospectiva coordinaban la logística, organizaban los cables para la proyección y la única persona del sector leía en su puesto atentamente un documento que contenía un mapa conceptual titulado "Árbol del Problema". En ese momento llegaron tres mujeres Trans y dos personas pertenecientes a la comunidad gay, uno de ellos era ciego.

Se inició la reunión con la presentación del nuevo monitor encargado de registrar los datos de todos, donde manifestó que estaba encantado de trabajar con el sector, pero muy apenado declaró su orientación heterosexual. Creo que eso sobraba, teniendo en cuenta que se llevaba casi una hora de retraso. Después de esto, se empezó haciendo un recuento de la anterior cita, señalándose además que la Mesa de Deporte y de Vivienda no había aportado nada. Ante esto, se procedió a aprobar la agenda para ese día. En esta tarea Prospectiva no muestra una diapositiva con el orden del día, sólo lo comenta, dificultándoseles a los presentes recordarlo, y así se aprueba el orden del día, alguien del sector dice irónicamente "si querido profesor". Después de esto uno de los funcionarios procedió a leer el acta basada en la reunión anterior. La lectura se le hizo eterna al grupo de asistentes, pues esta registraba cada unas de las intervenciones de los participantes, especificando con citas textuales, aunque se escuchara una voz de una de las personas del sector negando lo que ahi se señalaba de sus palabras. El acta parecía contener meros registros de palabras y no apuntalaba un marco lógico y de coherencia que lográramos discernir como oyentes. Al terminar la lectura, la mujer Trans que generalmente se tomaba la vocería apuntó comentarios y recomendaciones sobre la misma- "eso debe recoger todo, se nota que eso es copypaste de lo que yo le mandé, no se ve el interés porque un acta es la constancia de un proceso legal y el acta es la garantía que 


\begin{abstract}
nos sostiene a nosotras como asistentes-concluye diciendo - "Créanme que no es mi interés boicotear. Pero esta acta está perdida. Exijo respeto con la poca gente que está aquí sentada”, después de esto ella se hizo afuera del salón para calmarse y fumarse un cigarrillo. La mayoría de los asistentes estuvieron de acuerdo con esto y el acta no fue aprobada, sumado a que la persona gay ciega, le hizo una aclaración sobre el contenido del lenguaje en su discurso, pues según él actualmente ya no debe decirse personas en condición de discapacidad, sino que deben referenciarse solamente como discapacitadas. Seguidamente Prospectiva presenta una revisión sobre las políticas públicas y afirma que la base esencial de la misma debe ser la cultura. Después de esta intervención el debate se fue alargando, mientras que las mujeres Trans que se había tomado la vocería comentaba “ ¿Esto es una recocha? Debería haber un mejor manejo del moderador. ¿Quiero ver inclusión en todo! Voy a estar pendiente y meterle la cancha a esto"]
\end{abstract}

Observamos que en este escenario se presentaban más mujeres Trans que en Diplomado. Una de ellas tomaba la vocería muy vehementemente. Había personas que nunca se encontraban en el Diplomado y estaban participando en las mesas, como una persona discapacitada. Todos hablaban y opinaban sobre la política pública, pero cada intervención de los participantes era "acomodada" a la interpretación de uno de los miembros de Prospectiva, el cual no lograba tener un lenguaje asertivo y una actitud incluyente. Al parecer, ya se contaban con directrices, las cuales se encontraban representadas en un árbol de ideas, en el que se exponían los puntos que debían ser desarrollados. Nos pareció que paradójicamente, al ser tan pocas sesiones, el árbol ya trazaba una línea definitoria que daba cuenta de un proceso en el que se definían las prioridades de la población. La forma en que se abordaban los temas era un poco apresurado, claramente cuatro reuniones para hablar de siete mesas de trabajo con once temáticas, resultaba comprometedor, lo que no permitía abordar con profundidad los temas, teniendo en cuenta la prioridad que Hebert Pérez recalcaba sobre los asuntos de salud, incluidos en el Plan Decenal y justicia. El tema de justicia en una de las mesas en las que participamos se trató muy superficialmente, y por contratiempos se pasó a otro tema sin mayor discusión, los únicos aportes importes se hicieron por parte una activista mujer Trans que manifestó la necesidad de su población por un debido proceso y respeto a sus derechos. 
El interés de Prospectiva de presentar la cultura como el eje articulador de la política pública, parece quedarse en la mención. A pesar de que se presentó sobre la mesa la preocupación por informar a la comunidad en general de la política pública, de ejecutar acciones efectivas que intentaran socavar de raíz la homogeneización y estigmatización imbricada en la sociedad caleña, sus funcionarios no demostraron el interés de apropiarse del discurso, pues jocosamente uno de los funcionarios que cuenta con más responsabilidades dentro del contrato, al terminar una de las sesiones nos expresaba "así estas locas pelean" con el propósito posiblemente de contextualizarnos sobre lo que ocurría, de ahí que sean continuamente cuestionados. Por otro lado, la consecución de las labores de las mesas las lideraban personas del sector mismo, los contactos, las reuniones y lugares de encuentro corrían por cuenta de sus espacios libres entre la semana, las mesas de deporte y vivienda no lograron reunirse por fallas en la logística y comunicación entre los pares, pero esas fallas nunca se identificaron por parte de la institucionalidad, que contaba con una base de datos de todos los asistentes. Tal y como lo mencionó el representante de la Revista Ambición, las mesas se pensaron como un proceso a través del cual cada grupo correspondiente a ciertas mesas trabajara para reunir la información necesaria para los insumos, lo cual nunca dio resultado, empezando por la falta de comunicación entre los mismos participantes y la entidad y ellos, seguido del reducido grupo de personas participantes del proceso seguido de la imposibilidad de que los mismos participantes hicieran su propio trabajo de campo, teniendo en cuenta sus respectivas ocupaciones e intereses.

Las mesas concluyeron antes que el Diplomado y según algunos de los últimos y pocos asistentes, las reuniones al final se tornaron agresivas y terminaron en fuertes discusiones. Muy poco se sabe de en qué terminaron las mesas de trabajo, debido en primera instancia, al reducido número de asistentes y probablemente, a que estos últimos solo lo recuerden con aversión por lo sucedido el último día, mientras que otros lo pasan por alto y fingen que nada paso. El resultado de todo, es la nulidad del Acuerdo que ya no alcanza a ser aprobado para este año y que poco se revisó. Fue enviado a todos los asistentes faltando pocos días para el cierre de las correcciones y las cosas quedaron tal y como se estipuló en el documento. La duda que nos queda es si realmente el documento es producto de todo el proceso, esto teniendo en cuenta lo caóticas que fueron tanto las mesas como algunas sesiones del diplomado y los desacuerdos entre las partes. 
Es claro que hay un interés por parte de la alcaldía de desarrollar una política pública para el sector, y que esto se ha ido desarrollando gracias a la labor de la Secretaría de Bienestar Social y Desarrollo, que francamente independientemente de los tropiezos, la entidad como tal ha dado la cara y ha tenido el interes en la formulación pero en efecto, "eso no indica que puede hacer una política pública a la carrera sencillamente porque se le viene la ley de garantías y necesitan sacar ese proceso. Supongo que dijeron- O firmamos el proyecto ahora o se nos congela por la ley de garantías y ya no vamos a poder sacar esa platica y ese resultado (punto a favor dela oficina pues hicieron el proceso) rapidito sacamos esto con Prospectiva" (Entrevista a Representante de la Revista Ambición, 17 de noviembre 2013).

\section{Conclusiones}

A lo largo de los tres meses, el proceso de la formulación de la política pública LGBT local estuvo agitado por debates sobre la legitimidad de los actores de la contratación, aparición de intereses contrapuestos entre personas del sector, ausencia de las organizaciones más representativas y sesiones de clase descontextualizadas de la realidad de un sector demandante, el cual se hizo poco partícipe dentro de las reglas de juego.

Podríamos decir que contrario a lo que se pretendió inicialmente, una política incluyente con enfoque diferencial, de carácter bottom up si se quiere pensar, junto a un Diplomado de puertas abiertas, el resultado fue una política pública de carácter tradicional y tecnocrático top down que tiene un componente de exclusión, por ser un proceso vertical. En este caso, Prospectiva actúa como el funcionario que tiene que cumplir con unas metas, un contrato, sin tener serias consideraciones acerca de la comunidad, sin entender a profundidad el problema que se supone van a solucionar. Esto se debe a que han omitido ciertas realidades del sector/comunidad con la que están trabajando, hecho que se manifiesta en las actitudes y comentarios hechos por algunos miembros de Prospectiva que pretendieron trabajar con las pocas personas que hacían parte del Diplomado y las mesas de trabajo y prescindieron de la búsqueda de una solución a la falta de participación por parte de la comunidad LGBTI. Ello también es resultado de la ausencia de una participación de la población "que le de vida al proyecto y la lleve más allá del papel" (Arroyave, 2011:97). Hecho que podría explicarse con el desgaste de los asistentes del Diplomado y las Mesas 
para la construcción de la política pública, tanto por continuas fallas de la institucionalidad como por la falta de articulación entre los miembros del sector, que constantemente deben moverse en la compleja distinción entre la reivindicación de sus derechos y la asunción de responsabilidades como ciudadano.

Un proceso democrático y participativo debe velar por la constante legitimación de los involucrados en acciones como la efectiva transmisión de la información, alternativas de solución, actitudes más receptivas hacia la población, aclaración de las posibilidades y sensatez de cumplir con la agenda consensuada. Sin embargo, se observó que hay una carencia en la persuasión, comunicación y confianza entre los actores implicados. La articulación de las demandas con las herramientas que tiene el gobierno para generar la política no se ha dado y contrario a lo esperado, el resultado hasta ahora es la incertidumbre, el rechazo, un proceso con muchas trabas y disgustos que posiblemente no se concluya en tres meses como se esperaba. Tiempo que consideramos corto, comparado con la construcción de otras políticas públicas y mucho más teniendo en cuenta las complicaciones en términos de participación ya mencionados, sumado a que la política pública tiene miras hacia diez años de acciones locales en pro de la comunidad LGBT.

Por otro lado, la usual agresividad del sector, se debe en parte a la experiencia histórica de rechazo y vulneración de sus derechos y a la precaria reflexión que se hace sobre él, pues los estereotipos refuerzan imaginarios monolíticos de homogeneización que nos sucumbe en un velo de ignorancia. Aún así, es importante anotar que en un proceso tan complejo y crucial, ese tipo de comportamientos alteran significativamente el proceso e incluso lo complican, por ello no debe ser visto como justificado necesariamente. Hay un compromiso implícito dentro de lo anterior y es el esclarecimiento de los derechos y deberes de la comunidad LGBT, una delgada línea en donde los derechos deben ser exigidos de manera activa pero no agreste y los deberes deben estar en concordancia con el discurso de inclusión que estas personas promulgan hacia personas heterosexuales, con identidad fluida o cualquiera diferente a ellos y ellas. También hay un compromiso por parte del gobierno local que debe hacer frente a uno de los desafíos más grandes de las mesas, la cultura conjunta a las campañas de sensibilización y educación que contribuirían a la consecución de una ciudadanía más inclusiva, respetuosa, consciente de sus derechos y deberes y responsable con el resto de la sociedad. De manera que, avanzar 
hacia una política pública más efectiva supondría dar paso a una ciudadanía y a una cultura de participación junto a un involucramiento y seguimiento más cercano por parte de los actores estatales interesados en la política pública. Lo cual debe contrarrestar y contribuir a dar fin a la "injerencia de los actores políticos y sus intereses particulares que vician la formulación de las políticas y las decisiones públicas, desconociendo las prioridades en relación con el bienestar social de los ciudadanos, a los cuales se debería dirigir las políticas públicas" (Mejía, 2012:156).

De igual forma, no pretendemos afirmar que el Instituto Prospectiva no sea una institución idónea para la formulación de ésta política pública; sin embargo, concordamos con algunos entrevistados y participantes de la política a cerca de la importancia de incluir organizaciones que conocen del tema en la formulación de la política, la sinergia entre las organizaciones, líderes, liderezas, activistas, también junto a Prospectiva, pues daría resultado a una política pública mucho más inclusiva, que tuviera en cuenta las verdaderas necesidades de la población, atendiéndolos desde la raíz con la experticia de los participantes. Como lo señalaba uno de nuestros entrevistado:

"El Estado habla un solo idioma que es el idioma de la ciudadanía y esa es una de las cosas que ellos no han logrado entender. Pero de cierta manera lo entienden porque también se han prestado a esos procesos. Por ejemplo ustedes ven que los lineamientos que se hicieron son muy interesantes, son textos académicos gigantes, una producción intelectual envidiable (...) pero al tratar de poner en números el sufrimiento de las mujeres Trans (...) al hacer esa traducción fallan, porque el Estado no está interesado, porque perdería su esencia en decir: venga a usted yo le doy un trato diferencial" (Entrevista a Andrés Felipe Castelar, 12 de Octubre 2013)

El proceso hubiera podido ser ameno si en vez de confrontarse unos a otros, se hubiera establecido una alianza, un espacio de diálogo, deliberativo-pero no en exceso, más democrático que permitiera abordar las diferentes temáticas sin tener que preocuparse por la representatividad. Que a su vez permitiera a los funcionarios entender las problemáticas del sector, pero a manera complementaria de un extenso trabajo de campo que se ocupara de ello, expresado en insumos de las mesas de trabajo, pues en realidad fallaron a la 
hora de recoger los insumos suficientes y traducir las demandas sociales en unos inputs entendibles y procesables para el Estado.

El Instituto Prospectiva tuvo muchas dificultades durante el proceso, debió preparar un equipo que le garantizara a la entidad un mejor manejo del tema LGBT, el cual en su totalidad estuviera realmente dispuesto a lidiar con la sensibilidad del grupo en cuestión, sus demandas, críticas, para evitar el tipo de comentarios groseros y actitudes hostiles que dieron origen a los roces y problemas tanto en el Diplomado como en las mesas de trabajo. Dicho conocimiento previo habría sido un pilar para la consolidación de lazos de confianza, horizontalidad del proceso, entendimiento entre las partes, consenso y se pudo haber evitado el intercambio indistintamente del título de la política entre Política pública LGBTI, Política pública de Diversidad Sexual y de Género y Política pública para personas en Condición de Diversidad Sexual y de Género. De ahí que, el nombre de la política debería ser reconsiderado, teniendo en cuenta las implicaciones que tiene el uso de la palabra condición para referenciar a una población en especial. Ante el Estado esta población resulta estar en una posición de víctima, vulnerabilidad y minoría, que en parte ha sido producto de las acciones de la misma población, no obstante, esta no debe constituir la razón de uso del término condición dentro de la formulación de la política pública, incluso se predispone el proyecto, donde las personas probablemente serán tratadas como personas inferiores $\mathrm{y} / \mathrm{o}$ enfermas.

Otro punto a tener consideración es la incidencia que ha tenido la voz de los hombres homosexuales tanto en el Diplomado como en las mesas, y de las mujeres trans especialmente en las mesas de trabajo. Contradictoriamente las personas que más asistieron al diplomado eran mujeres, heterosexuales además, pero los que más participaban eran hombres homosexuales, y solo había entre dos o tres mujeres trans. Nos preguntamos qué pasó con las mujeres lesbianas, personas bisexuales, hombres trans y ni hablar de los intersexuales, de quienes nunca tuvimos conocimiento en todo el proceso del Diplomado, y por qué razón en las mesas personas como Andrea y otras dos mujeres trans si registraban mayor participación.

Finalmente, encontramos la trascendencia de una efectiva divulgación de la información política pública, no solo por parte de Prospectiva sino también por parte de la Secretaría. El formato virtual de la información no basta, también otro tipo de pronunciamientos 
contribuirían en otorgarle legitimación al proceso, como la generación de espacios de integración, que pudieron evitar contratiempos y complicaciones iniciales. De igual forma, el tipo de difusión que se lleve a cabo con la política debería ser aprobada por quienes hicieron parte del proceso y no de funcionarios desentendidos sobre la definición de una identidad de género y/o una orientación sexual diversa. ${ }^{8}$ El Plan de Acuerdo que se logre establecer en los próximos días deberá afrontarse a la aprobación del Concejo, donde probablemente se desarrollarán modificaciones, luego gran parte del proceso podría perderse, pues en la última reunión de las mesas, desistieron varias personas del proceso y manifestaron tomar medidas correctivas, reduciéndose aún más la cantidad de personas encargadas de concretar la formulación de la política pública local, lo cual resulta ser un panorama desolador para las personas del sector y para una política pública "bottom up". Esta política pública probablemente solo siga el modelo tradicional tecnocrático y finalmente termine siendo formulada por los especialistas en políticas públicas, omita las necesidades de la comunidad y no resuelva el problema a profundidad, se convierta en un proceso vertical, donde la participación termine siendo mínima y la articulación precaria.

"Si no se hace nada van a sacar lo que se les venga en gana, van a sacar una copia de lo de Buga, Bogotá y de lo del valle, recopilación de eso, que en ninguno se está implementando, pero Bogotá es otra realidad. En el peor de los casos la sacan. Si las cosas están ahi se pueden utilizar, pero que hayan sido bien elaboradas. Yo estoy descalificándola ya, el proceso no ha sido el correcto va a seguir siendo una política de pacotilla. Tenerla escrita y que la secretarias responsables de todo eso la dejen pasar" (Entrevista a Representante de Revista Ambición, 17 de noviembre de 2013).

8 "Cuando ha circulado lo que paso en cada mesa? Ni siquiera pudieron sacar varias mesas porque no había la suficiente gente que aportara a este proceso. No hay una comunicación real. Si se hace un proceso, se hace un acta, de esa acta es que salen los insumos. Esa acta se debe circularla entre todos los participantes y las otras personas que puedan interesarles y decir- esto fue lo que se dijo-. Pero si sencillamente se formula un documento aparte, en privado, no se está haciendo un proceso público. Están acomodando la información como se les antoja. No hay una base real de lo que están haciendo ¿Por qué tengo que mandar correos cerrados, con copia oculta y no permiten que vos des tu opinión abierta sobre lo que te están mandando? No tiene lógica. Yo necesito conocer la opinión de los demás para poderla refutar o para darle la razón. Se supone que desde la primera reunión de todos íbamos a aportar, cada uno en su casa -sino podía ir-, tenía que reconocer lo que se iba a aportar, nunca llegaba nada" (Entrevista a Representante de Revista Ambición, 17 de noviembre de 2013) 
Prospectiva debió haber estado en la capacidad de darle un giro a su accionar para ganarse la confianza de los participantes de la política y generar un espacio de mediación mucho más ameno, no utilizar modelos de política pública y adicionarle los componentes necesarios para diseñar una "nueva" política pública con el componente de diversidad sexual y de género. Hoy probablemente ya sea muy tarde para generar los cambios necesarios. Y quien sabe que logre llegar al Concejo, si llega a ser aprobada es probable que sea recortada, aún si no logra ser una política diseñada para las necesidades del sector, carente de legitimidad, porque no fue realizada por ell@s mism@s, entre otros. La responsabilidad de una política exitosa o no, no reside sobre algún actor en particular, Prospectiva como contratista habrá cumplido sus objetivos del contrato, entregará sus productos a la Secretaría y cobrará. Los asistentes tendrán sus diplomas, otros se quedaran con la intriga de qué pasara con el proceso y sus criticas habrán hecho algún efecto, las mesas resolverán la mitad o ni siquiera la mitad de las necesidades del sector, y la tarea de velar por su cumplimiento no encuentra directos responsables.

\section{Referencias}

Agarrones por política pública de la comunidad Lgtbi en Cali (2013,9 de Octubre) El Tiempo. Recuperado de http://goo.gl/LUCnwB

Alcaldía Mayor de Bogotá (2011) Balances y perspectivas: Política Pública para la Garantía Plena de los Derechos de las Personas Lesbianas, Gays, Bisexuales y Transgeneristas -LGBT-y sobre Identidades de Género y Orientaciones Sexuales en el Distrito Capital (pp.1-40) Bogotá:Alcaldía Mayor de Bogotá.

Alcaldía municipal de Santiago de Cali (2010). Lineamientos de la Política Pública para población en contexto de Diversidad Sexual y Géneros en el municipio de Santiago de Cali (pp.1-117) Cali: Alcaldía municipal de Santiago de Cali

Arroyave, S. (2011) Las políticas públicas en Colombia. Insuficiencias y desafíos.. Revista del Departamento de Ciencia Política. FORUM Nro. 1 Enero - Julio (pp. 1-17) Medellín : Universidad Nacional

Buriticá, I. C. (2010) Las Políticas Públicas Mujer y Géneros y LGBT y el caso de las transgeneristas. En La manzana de la discordia, 5(1), pp. 35-43. Bogotá: Universidad Nacional 
Cano, L. (2009). De la adopción a la adaptación de los discursos y métodos para el análisis de las políticas públicas en Colombia. En Análisis y evaluación de políticas públicas: debates y experiencias en Colombia (pp. 42-59). Bogotá: Universidad Nacional de Colombia.

Medellín es la primera ciudad en el país con política pública para el sector LGBT. (2011, 16 de abril). Caracol Radio. Recuperado de http://www.caracol.com.co/noticias/ regionales/medellin-es-la-primera-ciudad-en-el-pais-con-politica-publica-para-elsector-lgbt/20110416/nota/1456385.aspx

Mejía, J. (2012). Modelos de implementación de las políticas públicas en Colombia y su impacto en el bienestar social. En Analecta política, 2(3) (pp.141-164) Medellín: Universidad de Antioquia

Mérat, J. (2009). La evaluación frente a la política de la gestión pública. En Análisis y evaluación de políticas públicas: debates y experiencias en Colombia (pp. 8-14). Bogotá: Universidad Nacional de Colombia.

Revista Ambición. Página web. Recuperado de http://issuu.com/revsitaambicion/docs/ revistaambicion

Santa María Fundación. Página web (2005-2013). Recuperado de http://santamariafundacion.blogspot.com/

Sotomayor, S. (2013). Tras la siguiente pista: la configuración de los grupos LGBTI de la ciudad de Cali entre 1980 y 2013. Tesis de pregrado para optar por el título en Antropología (pp. 1-135) Cali: Universidad Icesi 